\title{
Coherent control of refractive indices
}

\author{
E. McCullough, M. Shapiro, ${ }^{*}$ and P. Brumer \\ Chemical Physics Theory Group, Department of Chemistry, University of Toronto, Toronto, Canada M5S 3H6
}

(Received 1 June 1999; published 6 March 2000)

\begin{abstract}
The application of coherent control to modify the real and imaginary parts of the refractive index $n$ of a material is described. Parameters to control the refractive index, and to minimize absorption, are identified. An application to gaseous $\mathrm{N}_{2}$ shows that extensive control over $n$ is possible.
\end{abstract}

PACS number(s): 42.50.Gy

There is considerable ongoing interest in manipulating quantum interference effects in order to alter the nonlinear optical properties of materials [1]. Here we show that one of the earliest coherent control scenarios [2,3] affords a versatile means of significantly increasing or decreasing the refractive index of molecules both off resonance and near resonance. Previous work on modifying the refractive index through coherence effects includes Refs. [4-6]. Harris and co-workers [4] focuses on broadband generation with concomitant reduction of the index of refraction in the case where electromagnetically induced transparency (EIT) is used to establish maximal two-level coherence (i.e., $\left|c_{1}\right|^{2}$ $=\left|c_{2}\right|^{2}=0.5$ below). Hau, Harris, and co-workers [5] invoke EIT in a Bose-Einstein condensate to modify the refractive index of sodium atoms by many orders of magnitude, slowing down the speed of light in this medium to $\approx 17 \mathrm{~m} / \mathrm{sec}$. Scully [6] focuses on increasing the refractive index, with low absorption, near resonances. By contrast, this work introduces new control parameters in the weak-field regime that allow for direct and extensive control over the real and imaginary parts of the refractive index both off resonance and near resonance.

We consider a system that has been prepared (e.g., by two-photon excitation) in a superposition state

$$
|\psi(t)\rangle=c_{1}\left|\phi_{1}\right\rangle \exp \left(-i E_{1} t / \hbar\right)+c_{2}\left|\phi_{2}\right\rangle \exp \left(-i E_{2} t / \hbar\right)
$$

of Hamiltonian eigenstates $\left|\phi_{i}\right\rangle$ of energy $E_{i}$ and that is then subjected to two cw fields of the form $(k=1,2)$

$$
\vec{\varepsilon}_{k}=\left[F_{k} \exp \left(i \omega_{k} t\right)+F_{k}^{*} \exp \left(-i \omega_{k} t\right)\right] \hat{\varepsilon}_{k} .
$$

where $\hat{\varepsilon}_{k}$ is the unit vector along $\vec{\varepsilon}_{k}$. We choose $\left(\omega_{2}-\omega_{1}\right)$ $=\left(E_{2}-E_{1}\right) / \hbar \equiv \Omega_{21}$, so that excitation of $\left|\phi_{1}\right\rangle$ by $\omega_{1}$ and of $\left|\phi_{2}\right\rangle$ by $\omega_{2}$ lead to the same energy $E=E_{1}+\hbar \omega_{1}=E_{2}$ $+\hbar \omega_{2}$ [2]. Our interest is in modifying the refractive index of the material at frequencies $\omega_{1}$ or $\omega_{2}$. We examine the case of isolated molecules, or molecules in a very dilute gas, where collisional relaxation and dephasing effects can be ignored. Further, the system and states are chosen so that the

\footnotetext{
*Permanent address: Chemical Physics Department, The Wiezmann Institute of Science, Rehovot, Israel.
}

radiative decay of $\left|\phi_{1}\right\rangle$ and $\left|\phi_{2}\right\rangle$ is negligibly small on the time scale associated with observing the effect described below.

An application of first-order perturbation theory gives the average value of the resultant system dipole moment as

$$
\langle\vec{\mu}\rangle=\sum_{m} c_{m}^{(1)}(t)\left(c_{1}^{*} \vec{\mu}_{1 m} e^{-i \Omega_{m 1} t}+c_{2}^{*} \vec{\mu}_{2 m} e^{-i \Omega_{m 2} t}\right)+c . c .,
$$

where $\vec{\mu}_{1 m}=\left\langle\phi_{1}|\vec{\mu}| \phi_{m}\right\rangle$ is the transition-dipole matrix element, and c.c. denotes the complex conjugate of the sum that precedes it and where the sum is over all $\left|\phi_{m}\right\rangle$. Here

$c_{m}^{(1)}(t)$

$$
\begin{aligned}
= & \frac{1}{\hbar} c_{1} \sum_{k=1}^{2} \mu_{m 1}^{\varepsilon_{k}}\left(F_{k}^{*} \frac{e^{i\left(\Omega_{m 1}-\omega_{k}\right) t}}{\Omega_{m 1}-\omega_{k}-i \gamma}+F_{k} \frac{e^{i\left(\Omega_{m 1}+\omega_{k}\right) t}}{\Omega_{m 1}+\omega_{k}-i \gamma}\right) \\
& +\frac{1}{\hbar} c_{2} \sum_{k=1}^{2} \mu_{m 2}^{\varepsilon_{k}}\left(F_{k}^{*} \frac{e^{i\left(\Omega_{m 2}-\omega_{k}\right) t}}{\Omega_{m 2}-\omega_{k}-i \gamma}\right. \\
& \left.+F_{k} \frac{e^{i\left(\Omega_{m 2}+\omega_{k}\right) t}}{\Omega_{m 2}+\omega_{k}-i \gamma}\right)
\end{aligned}
$$

where $\gamma$ is the average linewidth of bound levels that has been introduced phenomenologically, $\Omega_{m n}=\left(E_{m}-E_{n}\right) / \hbar$, $\vec{\mu}_{j m}=\left\langle\phi_{j}|\vec{\mu}| \phi_{m}\right\rangle$ and $\mu_{j m}^{\varepsilon_{k}}=\left\langle\phi_{j}\left|\vec{\mu} \cdot \hat{\varepsilon}_{k}\right| \phi_{m}\right\rangle$. Here we assume that (a) the cw fields are turned on at $t \rightarrow-\infty$, at which time the system is in its initial superposition state [Eq. (1)]; (b) the medium has no permanent dipole moment; and (c) $\left\langle\phi_{1}|\vec{\mu}| \phi_{2}\right\rangle=0$ due to parity requirements discussed later below. Inserting Eq. (4) in Eq. (3) gives 32 term contributions to $\langle\mu\rangle$ : half are proportional to $\left|c_{1}\right|^{2}$ or $\left|c_{2}\right|^{2}$ and hence involve the independent effects of $\omega_{1}$ and $\omega_{2}$ and half, proportional to $c_{i} c_{j}^{*}(i \neq j)$, are interference terms originating from the fact that we are dealing with an initial coherent superposition of $\left|\phi_{i}\right\rangle$. Of these 16 interference terms, eight do not oscillate with frequency $\omega_{1}$ or $\omega_{2}$ and hence do not contribute to the susceptibility $\chi(\omega)$ at these frequencies. Noting that $\vec{\mu}(\omega)=\left(\epsilon_{0} / \rho\right) \chi(\omega) \vec{F}(\omega) \exp (i \omega t)$, where $\epsilon_{0}$ is the permittivity of the vacuum, $\vec{F}(\omega)$ is the electric field at frequency $\omega$, and $\rho$ is the density of material, and using the expression for $\vec{\mu}(\omega)$ obtained as described above, we have 


$$
\begin{aligned}
& \epsilon_{0} \hbar \chi\left(\omega_{1}\right) / \rho \\
& =\sum_{m}\left|c_{1}\right|^{2} \vec{\mu}_{1 m} \vec{\mu}_{m 1}\left(\frac{1}{\Omega_{m 1}-\omega_{1}-i \gamma}+\frac{1}{\Omega_{m 1}+\omega_{1}-i \gamma}\right) \\
& \quad+\left|c_{2}\right|^{2} \vec{\mu}_{2 m} \vec{\mu}_{m 2}\left(\frac{1}{\Omega_{m 2}-\omega_{1}-i \gamma}+\frac{1}{\Omega_{m 2}+\omega_{1}-i \gamma}\right) \\
& \quad+\left(c_{1}^{*} c_{2} F_{2}^{*} / F_{1}^{*}\right)\left[\frac{\vec{\mu}_{1 m} \vec{\mu}_{m 2}}{\Omega_{m 1}-\omega_{1}-i \gamma}+\frac{\vec{\mu}_{m 2} \vec{\mu}_{1 m}}{\Omega_{m 2}+\omega_{1}-i \gamma}\right]
\end{aligned}
$$

$$
\begin{aligned}
& \epsilon_{0} \hbar \chi\left(\omega_{2}\right) / \rho \\
& =\sum_{m}\left|c_{1}\right|^{2} \vec{\mu}_{1 m} \vec{\mu}_{m 1}\left(\frac{1}{\Omega_{m 1}-\omega_{2}-i \gamma}+\frac{1}{\Omega_{m 1}+\omega_{2}-i \gamma}\right) \\
& \quad+\left|c_{2}\right|^{2} \vec{\mu}_{2 m} \vec{\mu}_{m 2}\left(\frac{1}{\Omega_{m 2}-\omega_{2}-i \gamma}+\frac{1}{\Omega_{m 2}+\omega_{2}-i \gamma}\right) \\
& \quad+\left(c_{1} c_{2}^{*} F_{1}^{*} / F_{2}^{*}\right)\left[\frac{\vec{\mu}_{2 m} \vec{\mu}_{m 1}}{\Omega_{m 2}-\omega_{2}-i \gamma}+\frac{\vec{\mu}_{m 1} \vec{\mu}_{2 m}}{\Omega_{m 1}+\omega_{2}-i \gamma}\right] .
\end{aligned}
$$

Below we assume that all of the incident light is linearly polarized along the $z$ axis and denote the laboratory $z z$ component of $\chi$ as $\chi_{z z}$. The desired index of refraction is given by $n\left(\omega_{i}\right)=\sqrt{1+\chi_{z z}\left(\omega_{i}\right)}$. Control over both the real and imaginary parts of $n(\omega)$ is of interest.

Examination of Eqs. (5) and (6) shows that $\chi(\omega)$ is comprised of two terms that are proportional to $\left|c_{i}\right|^{2}$ and that are associated with the traditional contribution, plus two fielddependent terms, proportional to $d_{i j}=c_{i}^{*} c_{j} F_{j}^{*} / F_{i}^{*}$, which results from the coherent excitation of $\left|\phi_{1}\right\rangle$ and $\left|\phi_{2}\right\rangle$ to the same total energy $E=E_{1}+\hbar \omega_{1}=E_{2}+\hbar \omega_{2}$. As a consequence, changing $d_{i j}$ alters the interference between excitation routes and allows for coherent control over the susceptibility, and hence control over the refractive index. Experimentally, this control is achieved by altering the parameters in the state preparation in order to affect $c_{1}, c_{2}$ and/or by varying the relative phase [7] and relative magnitude of $F_{1}, F_{2}$.

Three comments are in order. First, with $\left|\phi_{1}\right\rangle$ and $\left|\phi_{2}\right\rangle$ assumed to belong to the same electronic state, nonzero dipole products such as $\vec{\mu}_{1 m} \vec{\mu}_{m 2}$ require that $\left|\phi_{1}\right\rangle$ and $\left|\phi_{2}\right\rangle$ be of the same party. Thus, a superposition state that allows for control must be prepared via a parity-preserving process, e.g., by two-photon absorption. In this case, using a laser of frequency $\omega_{p}$ and of field amplitude $F_{p} \hat{\varepsilon}_{p}$, gives

$$
d_{12}=\sum_{n} \frac{\mu_{2 n}^{\varepsilon_{p}} \mu_{n 1}^{\varepsilon_{p}} F_{p}^{2 *} F_{2}^{*}}{\left(\Omega_{n 1}-\omega_{p}\right)(-i \gamma) F_{1}^{*}} .
$$

Here the $\varepsilon_{p}$ superscript denotes the component of the vector matrix element along $\hat{\varepsilon}_{p}$. Second, Eqs. (5) and (6) make clear that control over $\chi\left(\omega_{i}\right)$ is expected to be substantial if $F_{i} / F_{j}$ is large. However, under these circumstances control over $\chi\left(\omega_{j}\right)$ is minimal since the corresponding interference term is proportional to $F_{j} / F_{i}$. Hence, effective control over the refractive index is possible only at one of $\omega_{1}$ or $\omega_{2}$. Third, the assumption of perturbation theory implies that $\left|c_{2}\right|^{2}$ cannot exceed $\approx 0.2$, the value used below.

As an example of this general theory we consider modifying the refractive index of gaseous $\mathrm{N}_{2}$. Sample control results for $n(\omega)$, both off resonance and near resonance, are shown below. The off-resonance computations, carried out to convergence, included over 120 transition dipole matrix elements from rovibrational states of the ground $X \Sigma_{g}^{+}$electronic state to the vibrotational states of $b^{\prime 1} \Sigma_{u}^{+}, c^{\prime \prime} \Sigma_{u}^{+}$, $e^{\prime \prime} \Sigma_{u}^{+}, b^{\prime} \Pi_{u}, c^{\prime} \Pi_{u}$, and $0^{\prime} \Pi_{u}$ electronic states. The nearresonance computations approximate $\mathrm{N}_{2}$ as a three-level system. We assume the Condon approximation, use the electronic dipole transition matrix elements of Stahel et al. [8] with radial wave-function overlap calculated via uniform WKB, and include all vibrotational states needed for convergence. Control is shown as a function of the relative laser phase $\theta=2 \theta_{p}+\theta_{2}-\theta_{1}$ where $\theta_{p}$ is the phase of $F_{p}^{*}$ and $\theta_{i}(i=1,2)$ is the phase of $F_{i}^{*}$.

Figure 1 shows the dependence of the real [panel (a)] and imaginary [panel (b)] parts of $n\left(\omega_{1}\right)=n^{\prime}\left(\omega_{1}\right)+i n^{\prime \prime}\left(\omega_{1}\right)$, which are linked by causality, on $\left|F_{2} / F_{1}\right|$ for various different values of $\theta$. Results are shown for the nonresonant excitation of a superposition state comprised of $\left|\phi_{1}\right\rangle$ $=\left|v_{1}=0, J_{1}=0, M_{1}=0\right\rangle$ plus $\left|\phi_{2}\right\rangle=\left|v_{2}=0, J_{2}=2, M_{2}=0\right\rangle$, where $\left|c_{2}\right|^{2}=0.2, \omega_{1}=3 \times 10^{15} \mathrm{~Hz}$, and $\omega_{2}=2.99775 \times 10^{15}$ $\mathrm{Hz}$. The quantities $\left(v_{i}, J_{i}, M_{i}\right)$ denote quantum numbers for vibration, rotation, and for the projection of the angular momentum along the $z$ axis.

Consider first the case of $\theta=-\pi / 2$. Here $n^{\prime \prime}\left(\omega_{1}\right)=0$ (i.e., no absorption of the field) and $n^{\prime}\left(\omega_{1}\right)$ is seen to grow linearly on the log-log plot for $\left.\left|F_{2} / F_{1}\right|\right\rangle 10$; i.e., once the interference term in Eq. (6) dominates. Extensive control over $n^{\prime}$ is evident; e.g., $n^{\prime}$ has changed by well over $10 \%$ by $\left|F_{2} / F_{1}\right| \sim 10^{4}$. This is in sharp contrast with the tiny refractive-index changes associated, for example, with the optical Kerr effect or self-focusing (e.g., changing the index of refraction of $\mathrm{N}_{2}$ by as little as $10^{-6}$ via either of these effects requires laser intensities of $>10^{12} \mathrm{~W} / \mathrm{cm}^{2}$ ).

Figures 1(a) and 1(b) display a broad range of behavior of $n^{\prime}$ and $n^{\prime \prime}$. For example, for the case of $\theta=0$ and $\pi$ the $n^{\prime}$ increases for $\left|F_{2} / F_{1}\right|>1100$. For $\theta=0$ this increase is accompanied by positive $n^{\prime \prime}$, and hence by the absorption of the field. By contrast, the case of $\theta=\pi$ shows negative $n^{\prime \prime}$, i.e., the field is amplified. Also of interest is the case of $\theta=\pi / 2$, which shows rapidly decreasing $n^{\prime}$ with increasing $F_{2} / F_{1}$, accompanied by zero $n^{\prime \prime}$.

Additional computations indicated a strong dependence of control on $\left|c_{i}\right|$ and on the particular choice of $\left|\phi_{i}\right\rangle$. For example, results for initial superpositions comprised of $\left|\phi_{1}\right\rangle$ $=\left|v_{1}=0, J_{1}=0, M_{1}=0\right\rangle$ and $\left|\phi_{2}\right\rangle=\left|v_{2}, J_{2}, M_{2}=0\right\rangle \quad$ depended weakly on the choice of $J_{2}=0$ or $J_{2}=2$, but were heavily dependent on $v_{2}$. In particular, the larger the $v_{2}$, the smaller the $n^{\prime}$ at fixed $\left|F_{2} / F_{1}\right|$. This behavior is a direct consequence of the reduced size, for larger $v_{2}$, of the radial 

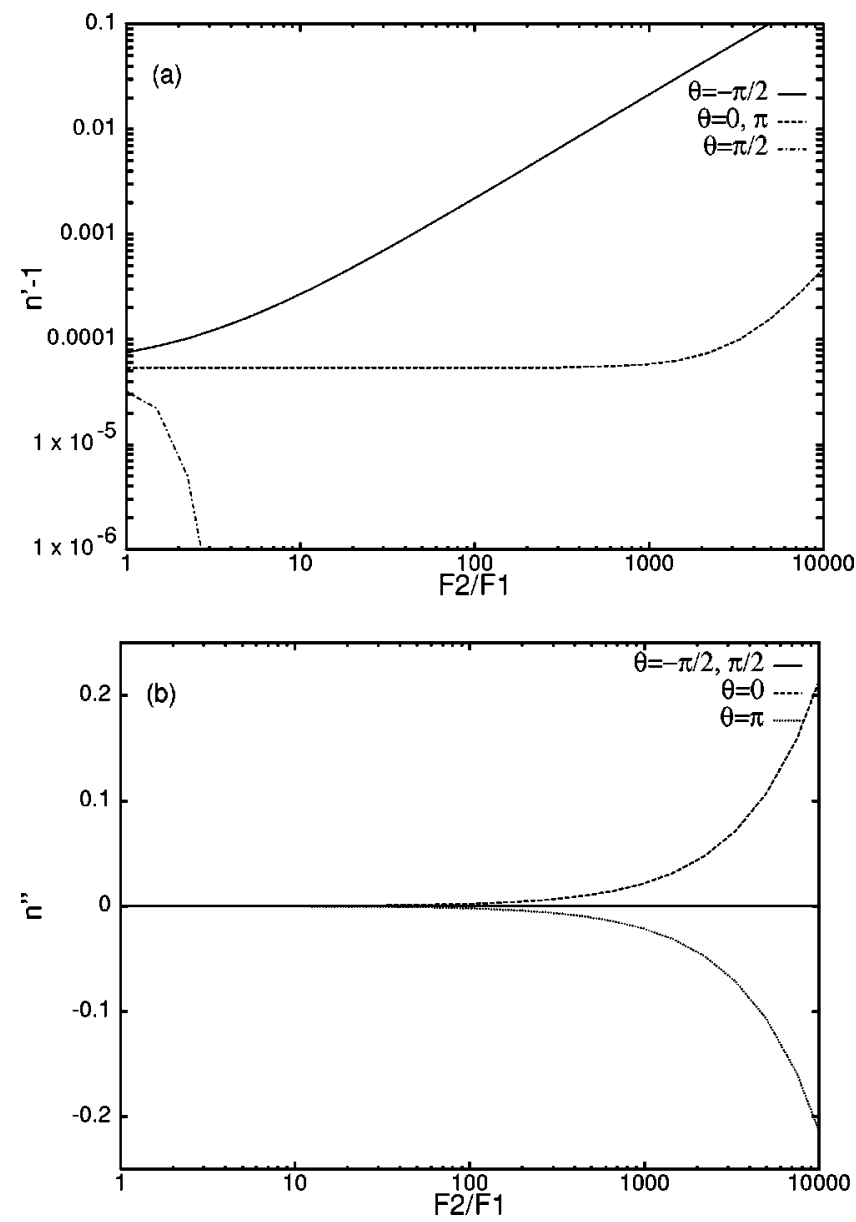

FIG. 1. Dependence of $n(\omega)$ on $F_{2} / F_{1}$ in $\mathrm{N}_{2}$ (in the superposition state described in the text) for different values of the relative laser phase $\theta$. (a) Real part $n^{\prime}(\omega)$ for $\theta=-\pi / 2$ (solid), $\theta=0$ and $\pi$ (dashed), and $\theta=\pi / 2$ (dot-dashed); (b) imaginary part $n^{\prime \prime}(\omega)$ for $\theta=-\pi / 2$ and $\pi / 2$ (solid), $\theta=0$ (dashed) and $\theta=\pi$ (dotted).

overlap matrix elements which, in turn, lower the value of dipole products such as $\vec{\mu}_{1 m} \vec{\mu}_{m 2}$, and hence reduce the interference contribution.

Altering $\left|c_{1}\right|$, either in a controlled fashion or, e.g., due to thermalization of population among various states, leads to a change in $n$. For example, Fig. 2 shows $n^{\prime}$ for the case of $\theta=-\pi / 2$, but where coefficients $\left|c_{1}\right|^{2}$ are extracted from state populations at $T=298^{\circ} \mathrm{K}$. Two examples are shown, the first is associated with having prepared a superposition of $\left|v_{1}=0, J_{1}=0, M_{1}=0\right\rangle$ with $\left|v_{2}=1, J_{2}=0, M_{2}=0\right\rangle$ and the second for a density matrix comprised of $\left(2 J_{1}+1\right)$ of superpositions of $\left|v_{1}=0, J_{1}=6, M_{i}\right\rangle$ with $\left|v_{2}=1, J_{2}=6, M_{i}\right\rangle, M_{i}$ $=-J_{1}$ to $J_{1}$, where $\left(v_{1}=0, J_{1}=6\right)$ is the most populated state at the given $T$. These superpositions (only a part of the full density matrix) would result from preparation via excitation [Eq. (7)] that is two-photon resonant with the energy spacing between the indicated levels. The results in Fig. 2 show a significant reduction in control over that shown in Fig. 1. Nonetheless, extensive control is predicted.

Consider now near-resonant excitation of the superposition state used in Fig. 1. Excitation with $\omega_{1}=1.900884279$ $\times 10^{16} \mathrm{~Hz}$, and $\omega_{2}=1.900659420 \times 10^{16} \mathrm{~Hz}$ excites the sys-

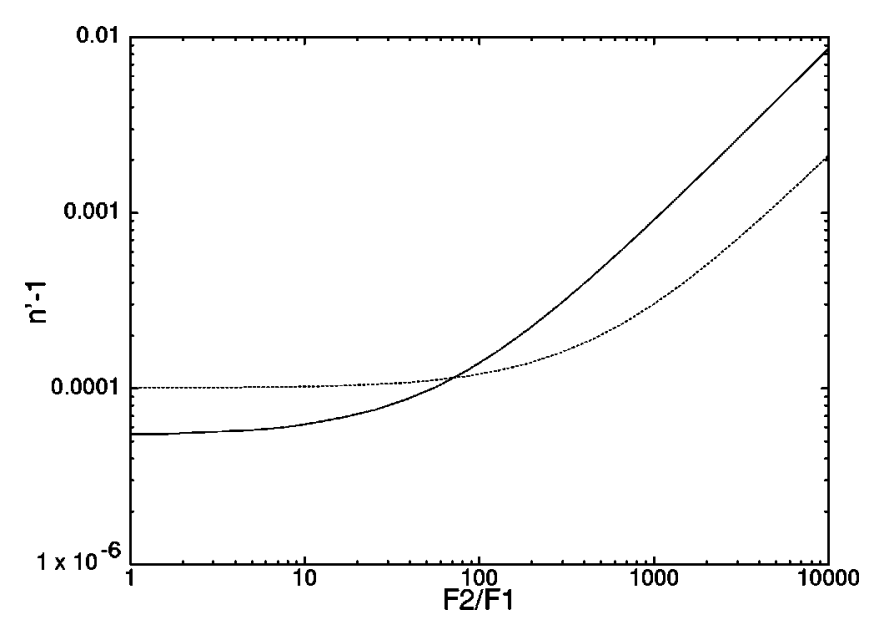

FIG. 2. Dependence of $n^{\prime}(\omega)$ on $F_{2} / F_{1}$ for a superposition of $\left|v_{1}=0, J_{1}=0, M_{1}=0\right\rangle$ with $\left|v_{2}=1, J_{2}=0, M_{2}=0\right\rangle$ where (solid line) molecules are in the ground state with $\left|c_{1}\right|^{2}$ determined by thermal populations at $T=298^{\circ} \mathrm{K}$ and (dashed line) for a density matrix comprised of superpositions of $\mid v_{1}=0, J_{1}=6, M_{1}=(-6$ to 6$\left.)\right\rangle$ with $\mid v_{2}=1, J_{2}=6, M_{2}=(-6$ to 6$\left.)\right\rangle$.
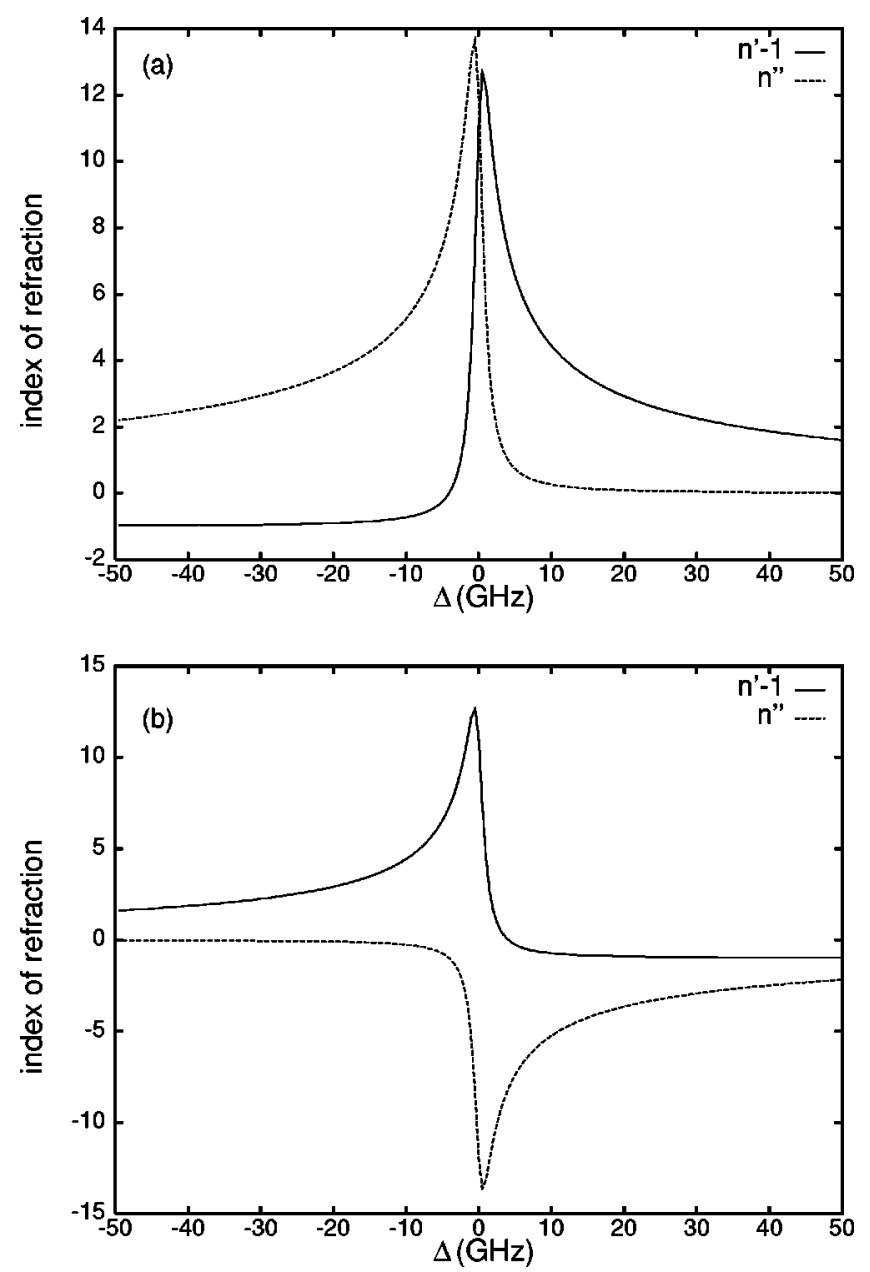

FIG. 3. Real and imaginary parts of the index of refraction of $\mathrm{N}_{2}$ as a function of the detuning $\Delta$ for the initial superposition state described in the text with $F_{2} / F_{1}=1000$. (a) $\theta=-\pi / 2$, (b) $\theta$ $=\pi / 2+10^{-6}$. 
tem, on resonance, to the $|v=0, J=1, M=0\rangle$ bound state of energy $E_{b}$ of the $b^{1} \Pi_{u}$ electronic state of $\mathrm{N}_{2}$. Consider then the refractive index as a function of detuning $\Delta$ (i.e., $E$ $\left.=E_{1}+\hbar \omega_{1}=E_{2}+\hbar \omega_{2}=E_{b}-\hbar \Delta\right)$. Figure 3(a) shows $n^{\prime}$ and $n^{\prime \prime}$ as a function of the detuning $\Delta$ for the choice $\theta=-\pi / 2, F_{2} / F_{1}=1000$. Here large values of the index of refraction are seen to be associated with negligible absorption at $\Delta>20 \mathrm{GHz}$, and the group velocity of light $v_{g}$ $=c /\left[n^{\prime}+\omega_{1} d n^{\prime} / d \omega_{1}\right]$ in this regime [5] can be estimated to be $150 \mathrm{~m} / \mathrm{sec}$. The sensitivity to the control parameters is evident by changing $\theta$ to $\pi / 2+10^{-6}$, shown in Fig. 3(b). Here $n^{\prime \prime}$ is negative, corresponding to amplification of the beam. One should note then that a rich range of behavior is possible in near-resonance cases as the control variables $d_{i j}$ are altered. Numerous examples are provided in Ref. [9].

In summary, this simple control scenario affords the possibility of a broad range of control over the refractive index. Applications to $\mathrm{N}_{2}$ show that the control range is extensive. The scenario discussed in this Rapid Communication, where a superposition of two levels is excited by two cw lasers, is just one of many possible coherent control scenarios that take advantage of quantum interference effects [3]. Other coherent control scenarios, as well as the possibility of lasing in the region of negative $n^{\prime \prime}$, are currently under investigation.

Support from the U.S. Office of Naval Research is gratefully acknowledged.
[1] See, e.g., M.O. Scully and M.S. Zubairy, Quantum Optics (Cambridge University Press, Cambridge, 1997).

[2] P. Brumer and M. Shapiro, Chem. Phys. Lett. 126, 541 (1986).

[3] For a recent review, see M. Shapiro and P. Brumer, J. Chem. Soc., Faraday Trans. 93, 1263 (1997).

[4] M. Jain, H. Xia, G.Y. Yin, A.J. Merriam, and S.E. Harris, Phys. Rev. Lett. 77, 4326 (1996); S.E. Harris and A.V. Sokolov, Phys. Rev. A 55, R4019 (1997).

[5] L.V. Hau, S.E. Harris, Z. Dutton, and C.H. Behroozi, Nature (London) 397, 594 (1999).

[6] M.O. Scully, Phys. Rev. Lett. 67, 1855 (1991); A.S. Zibrov,
M.D. Lukin, L. Hollberg, D.E. Nikonov, M.O. Scully, H.G. Robinson, and V.L. Velichansky, ibid. 76, 3935 (1996); M. Fleischhauer, C.H. Keitel, M.O. Scully, C. Su, B.T. Ulrich, and S-Y Zhu, Phys. Rev. A 46, 1468 (1992).

[7] C. Chen, Y-Y. Yin, and D.S. Elliott, Phys. Rev. Lett. 64, 507 (1990); S.M. Park, S-P. Lu, and R.J. Gordon, J. Chem. Phys. 94, 8622 (1991).

[8] D. Stahel, M. Leoni, and K. Dressler, J. Chem. Phys. 79, 2541 (1983).

[9] E. McCullough, M.Sc. dissertation, University of Toronto, 1997 (unpublished). 